\title{
Chapter 6 \\ The Importance of the Affective \\ Component of Movement in Action \\ Understanding
}

\author{
Giuseppe Di Cesare
}

\begin{abstract}
Social interactions require the ability to evaluate the attitudes of others according to the way in which actions are performed. For example, a hand gesture can be kind or vigorous or the tone of voice can be pleasant or rude providing information about the attitude of the agent. Daniel Stern called these aspects of social communication vitality forms. Vitality forms continuously pervade the life of individuals and play a fundamental role in social relations. Despite the importance of vitality forms, very little is known on their neural basis. The aim of the present chapter is to provide an overview of the neural substrates underpinning the encoding of these aspects of social communication. This chapter is organized in four sections. Section 6.1 describes the structural and functional domains of the insular cortex. Section 6.2 provides evidence that the dorso-central insula plays a central role in the perception and expression of action vitality forms. Section 6.3 demonstrates that the same insular sector is also involved in the perception of words conveying gentle and rude vitality forms. Finally, Sect. 6.4 discusses the important role of vitality forms in social interactions and proposes some future perspectives.
\end{abstract}

\subsection{Introduction}

When observing actions performed by others, we are able to understand the actiongoals as well as their intentions. These abilities are related to the existence of a basic brain mechanism known as "mirror mechanism" that transforms sensory representation of others' behavior into one's own motor representation of that behavior [12]. This mechanism is based on the activity of a distinct class of neurons that discharge both when individuals perform a goal directed action and when individuals observe another person performing the same action. Originally, mirror neurons were discovered in the ventral premotor cortex of the macaque monkey (area F5; di Pellegrino et al. [8]). Subsequently, the mirror mechanism has been found in humans in the

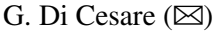

Istituto Italiano di Tecnologia, Genoa, Italy

e-mail: giuseppe.dicesare@iit.it 
parietal and premotor cortices as well as in anterior cingulate cortex [2] and in the anterior insula [16].

In addition to goal (what) and motor intention (why), there is another fundamental aspect of the action: the form (how). The aim of the present chapter is to focus on the action form highlighting its fundamental role in social communication. Indeed, during interpersonal relations, actions can be performed in different ways. For example, a hand shake can be gentle or vigorous, and a caress can be delicate or rushed communicating the positive or negative attitude of the agent. Similarly, words can be pronounced with a kind or unkind tone also conveying the agent's attitude. These different forms of communication have been named "vitality forms" by Stern [15]. Vitality forms continuously pervade the life of individuals characterizing their behaviors. The execution of vitality forms allows the agent to express his own mood/attitude, while the perception of vitality forms allows the receiver to understand the mood/attitude of others. For example, observing a person interacting with you, you may instantly understand if that person is glad or not and the same thing goes for words. Indeed, answering the phone, it is possible to understand how the other person feels by listening to the tone of voice. An interesting question is to investigate the neural correlates of these forms of communication. Results obtained in a functional magnetic resonance imaging study (fMRI) showed that the observation of actions performed with rude and gentle vitality forms produced the activation of a small part of the brain named dorso-central insula [3]. Most importantly, this brain area is activated not only during the perception of gentle or rude actions but also during their execution [6]. Thus, the activation of the same area for both the observation and execution of vitality forms strongly suggests the existence of a mirror mechanism for action vitality forms in the dorso-central insula. Differently from the mirror mechanism located in the parietal and frontal areas specific for the action goal understanding, the mirror mechanism located in the insula might allow one to express own mood/attitude and to understand those of others. It is important to note that, the same mechanism is also involved in the perception (listening) and expression of action verbs pronounced gently or rudely (speech vitality forms; Di Cesare et al. [4, 7]).

All these findings highlight that the insular cortex is the key node involved in the processing of vitality forms and suggest its plausible role in modulating the affective aspect of actions and words. The ability to express and recognize vitality forms allow people to be socially connected. Indeed, during interpersonal relations, the expression of vitality forms allows the agent to communicate by gestures or words his own affective state, while the perception of vitality forms allows the receiver to understand the positive or negative attitude of the agent and prepare an adequate motor response.

In this regard, vitality forms are a valuable feature of social communication useful to promote human-human and human-robot interactions.

The present chapter will provide an overview of action and speech vitality forms highlighting the neural substrates underpinning the encoding of these aspects of social communication. In particular, this chapter is subdivided into four sections devoted to the following topics: 
1. Structural and functional domains of the insula. In this first section, it will be illustrated and described the anatomical structure of the insula indicating the location of the dorso-central insula, which is involved in the processing of vitality forms.

2. The encoding of action vitality forms. The second section will present fMRI data showing that the dorso-central insula is active during the observation and the execution of action vitality forms.

3. The encoding of auditory vitality forms. The third section will describe fMRI data showing that listening to action verbs pronounced with gentle and rude vitality forms activates the dorso-central insula.

4. The role of vitality forms in social interactions. Finally, the fourth section will show behavioral data highlighting that, during social interactions, the vitality form expressed by the agent influences the subsequent motor response of the receiver.

\subsection{Structure and Function of the Insula}

In humans, the anatomical structure of the insula has been described for the first time by Johann Christian Reil (1809). It is a small part of the brain, located in both the left and right hemispheres in the depth of the Sylvian fissure (Fig. 6.1a). Anatomically, the insula is made up of anterior and posterior parts separated by the central insular sulcus (CIS). The anterior insula includes the anterior, middle, and posterior short gyri (asg, msg, psg), while the posterior insula includes the anterior and the posterior long gyri (alg, plg) (Fig. 6.1b). Being anatomically connected with the amygdala, thalamic nuclei, and with many other cortical areas, the insula is involved in several different functions, such as attention, pain, gustation, and the processing of emotions. On the basis of a meta-analysis carried out on 1768 functional neuroimaging studies, Kurth et al. [10] described the functional organization of the insular cortex. In particular, the authors identified four distinct functional domains in the insula: the sensory-motor (SM), the olfactory-gustatory (OG), the socio-emotional (SE), and the cognitive domain (CG) (Fig. 6.1c). In this chapter, it will be discussed the role of dorso-central insula (DCI), which is composed by the middle and posterior short gyri (msg and psg; Fig. 6.1b), in the encoding of action and speech vitality forms.

\subsection{The Encoding of Action Vitality Forms}

During social interactions, important information about others' behavior is carried out by the form of the action. Action vitality form describes "how" an action is performed, representing an important aspect that an observer may capture viewing an action performed by others. Differently from the action goal (what) and intention 


\section{Insular structure and functional domains}

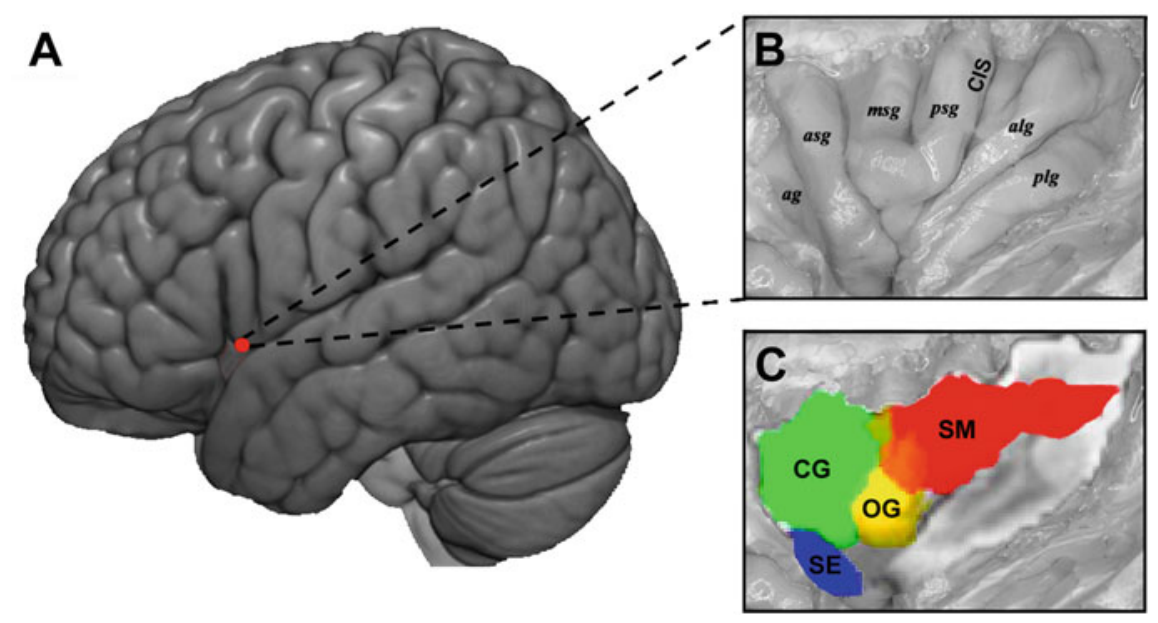

Fig. 6.1 Localization of the insula in the human brain (a). Anatomical structure of the insula (b): accessory gyrus (ag); anterior short gyrus (asg); middle short gyrus (msg); posterior short gyrus (psg); central insular sulcus (CIS); anterior long gyrus (alg); posterior long gyrus (plg). Functional domains identified in the right insular cortex: sensory-motor (red), olfactory-gustatory (yellow), socio-emotional (blue), and cognitive domain (green) (c). Figure adapted from Kurth et al. [10]

(why), vitality form (how) reflects the internal psychological state of the agent, providing also an appraisal of the affective quality underlying the relation between the agent and the action recipient [15]. In the first, fMRI study was investigated the neural correlates involved in the recognition of vitality forms. To this purpose, 19 healthy right-handed participants were presented with video clips lasting $3 \mathrm{~s}$ showing interactions between two actors that performed 4 actions without object (stroke the other actor's backhand, shake hands, clap hands, stop gesture; Fig. 6.2a) and 4 actions with object (pass a bottle, hand a cup, pass a ball, give a packet of crackers; Fig. 6.2b). Most importantly, each action was performed with a gentle or rude vitality form (Fig. 6.2c). During the fMRI experiment, participants were requested to pay attention either to the action goal (what task) or to the action vitality form (how task).

Results showed that the contrast between the two tasks (what vs. how) revealed activations for the what task, in the posterior parietal lobe and premotor cortex bilaterally, and in the caudal part of the inferior frontal gyrus of the left hemisphere (Fig. 6.3a). The opposite contrast (how vs. what) revealed a specific activation for the how task in the dorso-central insula of the right hemisphere (Fig. 6.3b). These data indicate that, paying attention to the action goal (what task) produces the activation of the parieto-frontal circuit classically involved in the action goal understanding [11]. In contrast, paying attention to the action vitality form (how task) produces the activation of the dorso-central insula. The main finding of this first fMRI study 

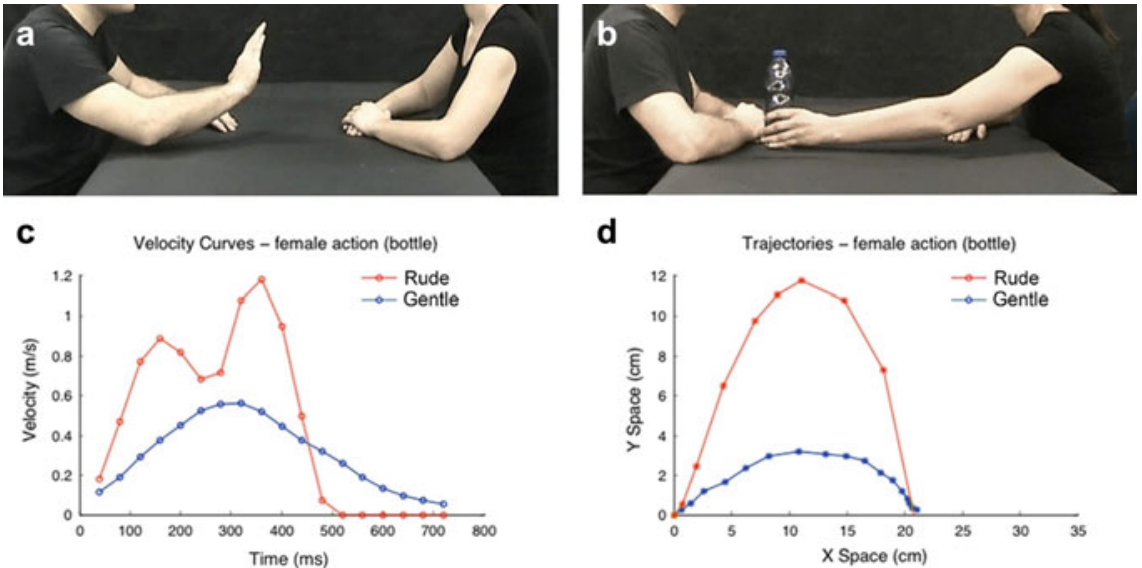

Fig. 6.2 Example of video clips observed by participants during the experiment. Frame representing an actor executing a stop gesture (a); frame representing an action with an object (passing a bottle; b). Velocity profiles (c), and trajectories (d) associated with one of the actions (passing a bottle) performed by the female actress with two vitality forms (rude: red line; gentle: blue line). As shown by graphs, the rude action was characterized by a hither velocity and a wider trajectory ( $Y$ space) than that observed for the gentle one. Figure adapted from Di Cesare et al. [3]

\section{A What vs. How}

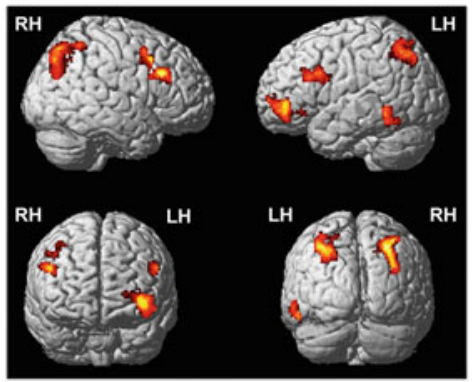

B How vs. What

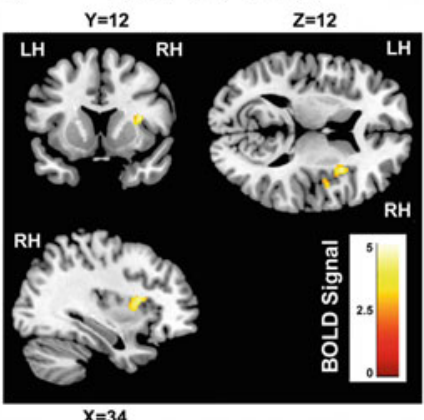

Fig. 6.3 Brain activations resulting from the direct contrasts what task versus how task (a) and how task versus what task (b). These activations are rendered into a standard MNI brain template (PFWE $<0.05$ at cluster level). $L H$ Left hemisphere; $R H$, right hemisphere. Figure adapted from Di Cesare et al. [3]

was the demonstration that, during action observation, the insula is the brain region involved in the processing of gentle and rude action vitality forms.

During social interactions, people not only observe vitality forms but also perform them. An interesting question is to investigate whether the dorso-central insula underlies both observation and execution of vitality forms. This issue was assessed in a subsequent fMRI study carried out on 15 healthy right-handed participants. 
In this experiment, participants were requested to perform three different tasks: observation (OBS), imagination (IMA), and execution (EXE). In the observation task, participants observed video clips showing an actor passing an object in a gentle and rude way (vitality form condition; Fig. 6.4a1-b1) or placing a small ball in a box (control condition; Fig. 6.4c1). In the vitality form condition, during the imagination and execution tasks participants were requested to imagine to pass an object toward the actor facing them (Fig. 6.4a2-b2) or to move the object in a rude (Fig. 6.4a3) or gentle way (Fig. 6.4b3). In contrast, in the control condition, participants were requested to imagine to place a small ball in the box (imagination task; Fig. 6.4c2) or to place it without any explicit vitality form (execution task; Fig. 6.4c3).

The results of the conjunction analysis showed that in all three tasks (observation, imagination, execution) for each condition (rude, gentle, Ctrl), there was a bilateral activation of the premotor and parietal cortices plus a strong activation of the left somatosensory cortex, motor cortex and the dorsal part of the cerebellum (Fig. 6.5). In addition, in rude and gentle conditions, there was also the activation of the middle temporal area, the inferior frontal gyrus and the posterior parietal cortex. Most

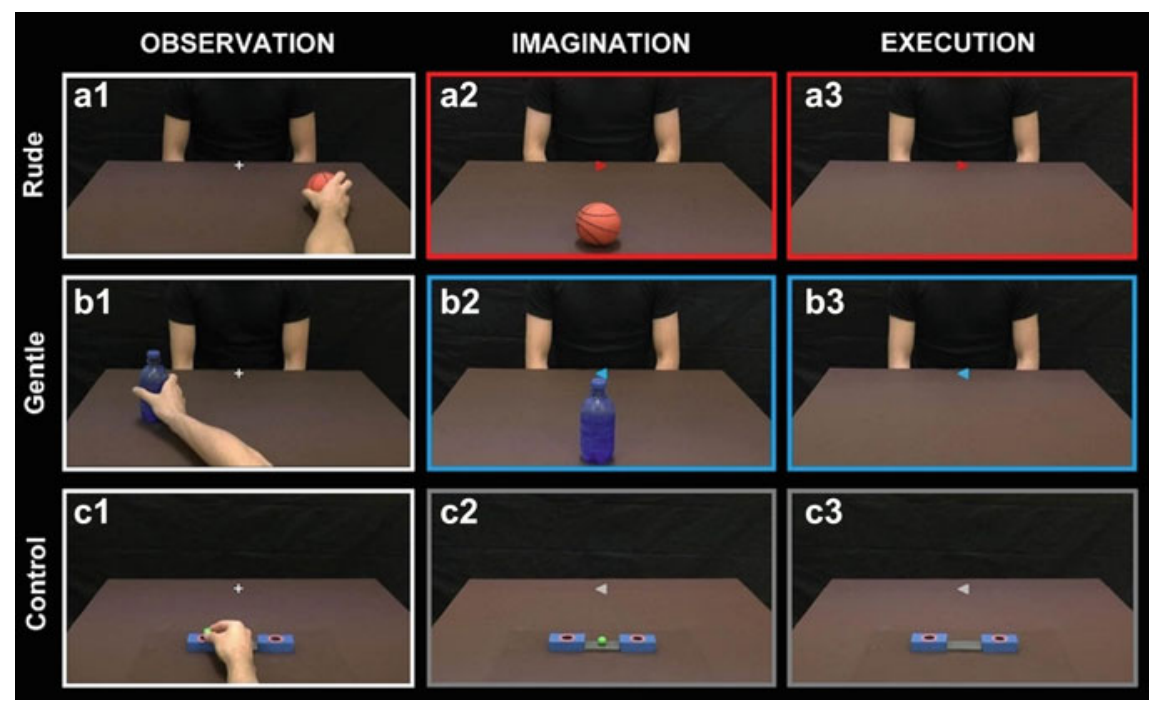

Fig. 6.4 Experimental design. Left column: Observation task. Participants observed the right hand of an actor moving an object in rightward (a1) or leftward (b1) directions. The observed action could be performed with a gentle or rude vitality form and the task request was to pay attention on the action vitality form. As a control participants observed the actor's hand placing a small ball in the right or left box (c1). Middle column: Imagination task. According with the edge screen color (red or blue), participants were requested to imagine themselves to pass an object toward another actor with a rude (red color; a2) or gentle (blue color; b2) vitality form. As a control the participants imagined to place a small ball in the right or left box (c2). Right column: Execution task. The participants moved a packet of crackers with a rude (red color; $\mathbf{a} 3$ ) or a gentle (blue color; b3) vitality form toward the actor facing them. As a control the participants had to place a small ball in the box (c3). Figure adapted from Di Cesare et al. [6] 


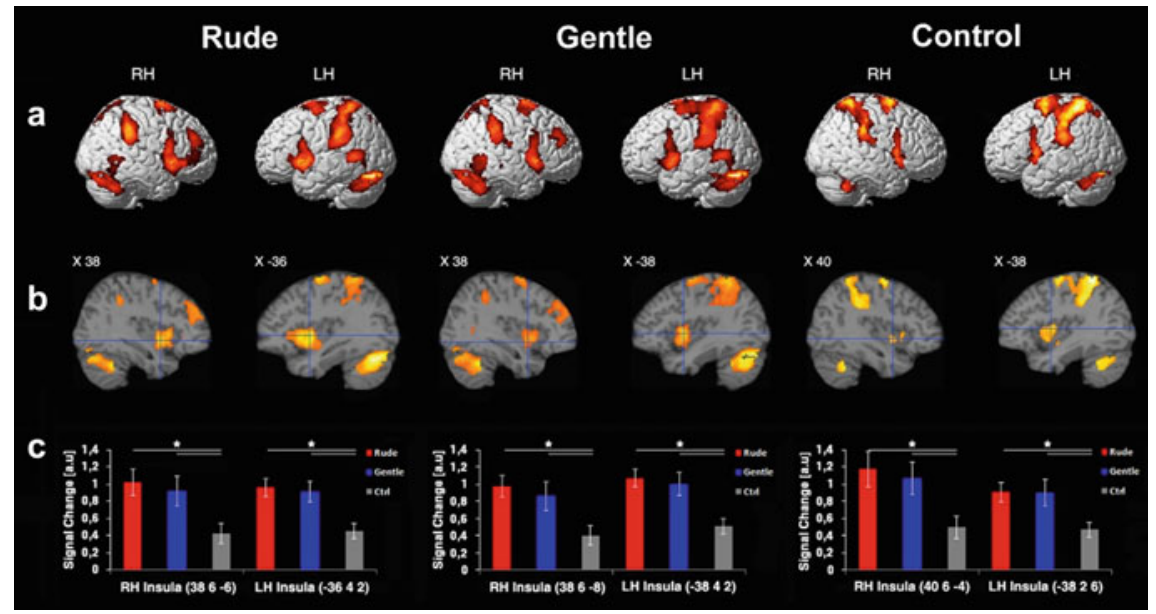

Fig. 6.5 Overlapping of areas activated in all three tasks (OBS, IMA, EXE). Lateral views of the brain activations obtained in the right and left hemispheres (a). Parasagittal sections showing the insular activations in the two hemispheres during the three tasks (b). These activations are rendered on a standard MNI brain template (PFWE $<0.05$ at cluster level). BOLD signal were extracted from six regions of interest (ROIs) created on the dorso-central insula. All ROIs were defined centering the sphere (radium $10 \mathrm{~mm}$ ) around the maxima of the functional maps resulting from a conjunction analysis of OBS, IMA, and EXE tasks. The horizontal lines indicate the comparisons between gentle, rude, and control conditions. Asterisks indicate significant differences $(p<0.05$, Bonferroni correction). Figure adapted from Di Cesare et al. [6]

importantly, this analysis revealed a selective activation of the dorso-central part of the insula when the action was observed, imagined to perform and performed with a gentle or rude vitality form (Fig. 6.5b, c).

The finding that the dorso-central insula is involved in both vitality form perception and expression suggests that neurons of this insular sector might be endowed with the mirror mechanism transforming visual representation of the perceived vitality forms in their motor representation. This view is in line with other fMRI findings demonstrating that the anterior sector of the insula is active during both the expression and recognition of disgust in others [16]. A similar matching mechanism is likely involved in feeling of pain and in recognizing it in other [13]. Thus, the anterior and dorso-central sectors of the insula, although underlying different functions appear to be both endowed with the mirror mechanism.

\subsection{The Encoding of Auditory Vitality Forms}

During social interactions, words may be pronounced in gentle or rude way conveying different vitality forms. Listening to different speech vitality forms allows 
the receiver to understand the positive or negative attitude of the speaker. For example, answering the phone, it is possible to understand how the other person feels by hearing the tone of voice. As described for the action, the speech vitality forms allow people to communicate their internal state and to understand those of others by modulating the tone of voice $[5,7]$. The ability to express and to understand the auditory vitality forms is already present in infants [14]. During mother-child interactions, the mother pronounces words by using a childish language. In particular, during the verbal communication with their children, mothers voluntary slow down the pronunciation of the verbal material adapting their language to the perceptive and expressive capacities of their children [1].

An interesting question is to understand whether the dorso-central insula, involved in the encoding of action vitality forms, is also involved in the encoding of speech vitality forms. In order to address this issue, an fMRI study was carried out on 16 healthy right-handed participants [4]. In particular, participants were presented with audio stimuli consisting of four Italian action verbs [Italian verbs: "dammi" (give), "prendi" (take), "tocca" (touch), "strappa" (tear)] pronounced by a male actor and a female actress. Most importantly, all the action verbs were pronounced using two different vitality forms: rude and gentle (vitality condition; Fig. 6.6a1-b1). For each action verb, two controls were presented: a robotic voice (robot condition) pronouncing the same action verbs as the actors; a scrambled version of the action verbs pronounced with gentle and rude vitality forms (scrambled VF condition). With regard to the robot condition, the robotic voice pronounced the same action verbs maintaining the meaning but not conveying any vitality form (Fig. 6.6a2-b2). In contrast, concerning the scrambled condition, the scrambled stimuli maintained the
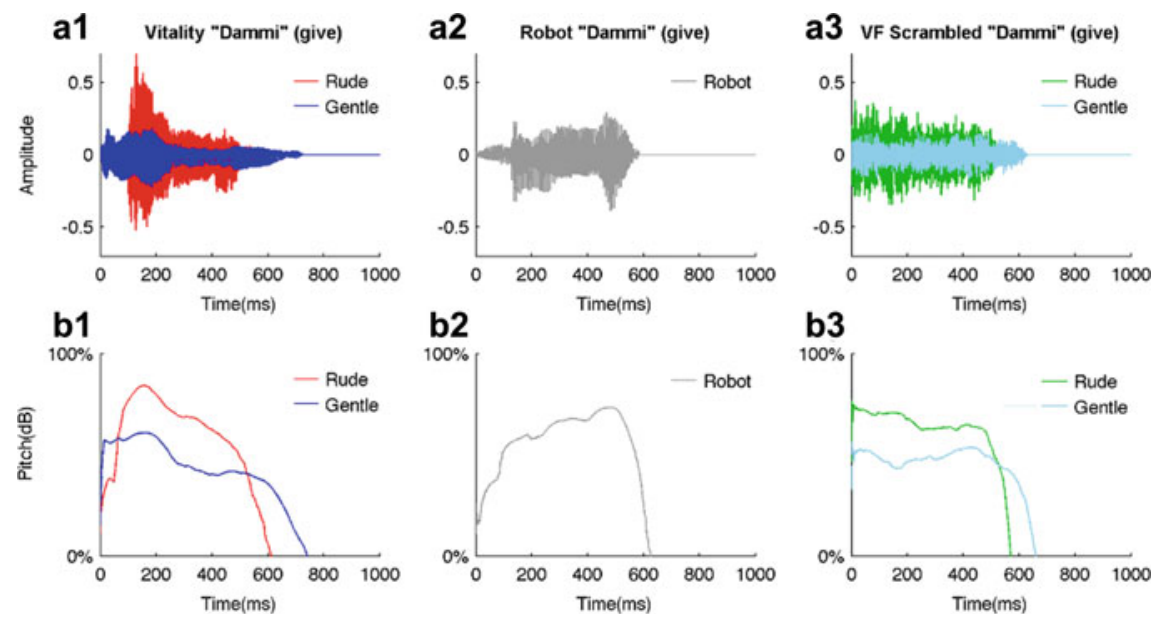

Fig. 6.6 Physical characteristics relative to the action verb "dammi" (give). Graphs a show the audio wave amplitude for all three categories [a1 vitality: rude (red color), gentle (blue color); $\mathbf{a} 2$ robot (gray color); a3 scrambled VF: rude (green color), gentle (cyan color)]. Graphs b show the sound intensity of each stimulus category. Figure adapted from Di Cesare et al. [4] 
physical properties of the stimuli (pitch, amplitude) but did not convey any meaning (Fig. 6.6a3-b3).

The results indicated that hearing vitality forms action verbs produced activations of the superior temporal gyrus, left inferior parietal lobule, left premotor, left prefrontal cortex, and posterior part of the inferior frontal gyrus plus a bilateral activation of the insula (Fig. 6.7a, left side). A very similar activation pattern was observed for the robot condition except for the insula activation (Fig. 6.7a, center). In contrast, listening to scrambled stimuli produced only the activation of the auditory temporal areas (Fig. 6.7a, right side). Most importantly, the direct contrasts vitality forms versus robot and vitality forms versus scrambled of revealed a significant activation of the left central part of the insula (Fig. 6.7b).

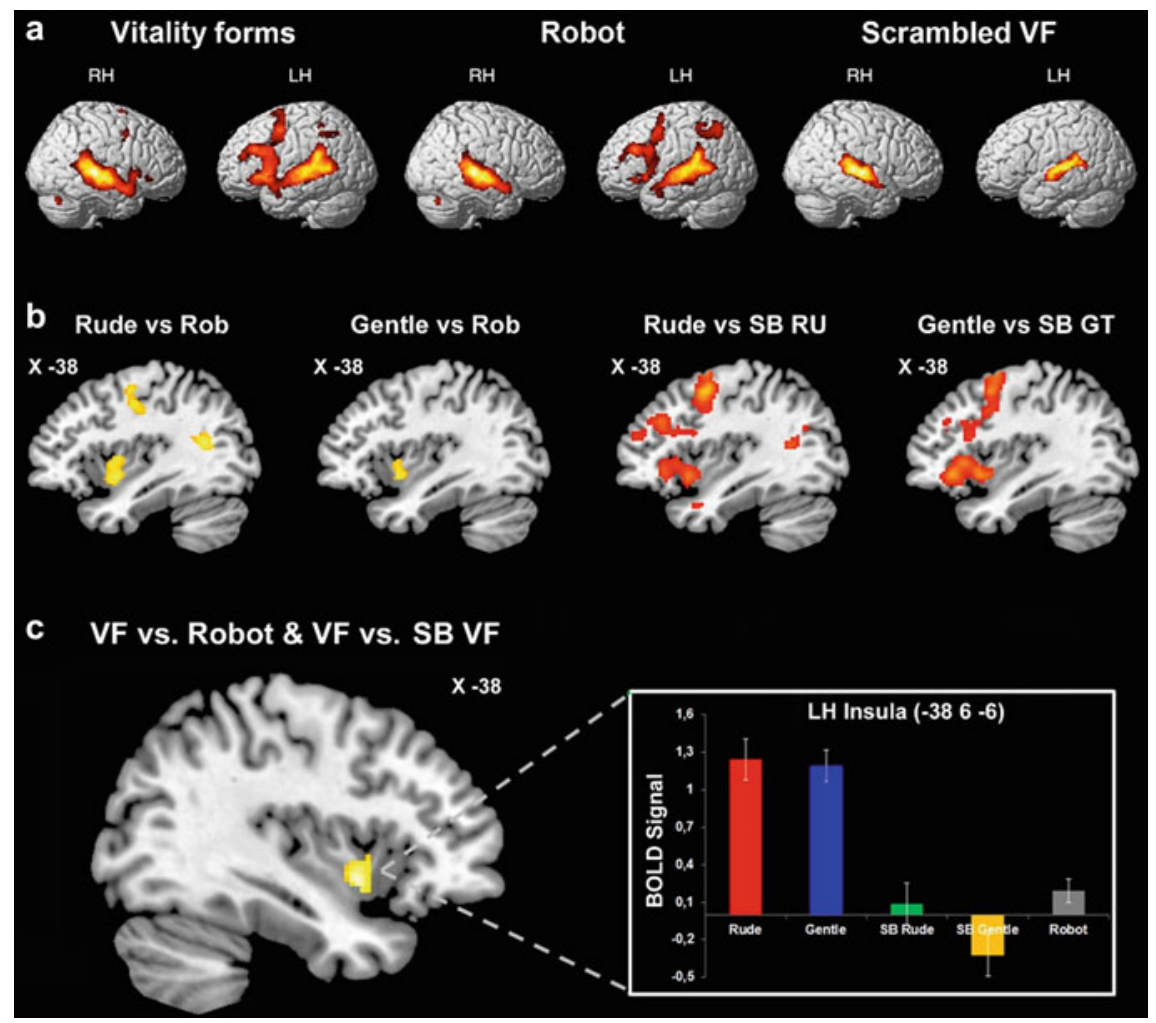

Fig. 6.7 Brain activations obtained in the hearing of different stimuli categories (a). Parasagittal sections showing the activations resulting from the contrast rude versus robot, gentle versus robot, rude versus scrambled rude; gentle versus scrambled gentle (b). Conjunction analysis evidences voxels activated in both contrasts vitality forms versus robot and vitality forms versus scrambled $v f$ (c; left side). BOLD signal recorded in the insular area highlighted from the conjunction analysis (c; right side). To avoid circular analysis, statistical comparison was not carried out between conditions [9]. Figure adapted from Di Cesare et al. [4] 
The finding that the dorso-central insula is activated during the hearing of vitality forms cannot be merely accounted for the meaning of action verbs. Indeed, although the robotic voice conveyed the same verbal message, the insula was activated only when participants listened to action verbs conveying vitality forms. Additionally, listening to scrambled stimuli, although the physical properties (intensity, frequency) were the same between scrambled and vitality forms conditions, it did not produce the activation of the insula excluding the possibility that the insular activity could be due to the physical properties of the auditory stimuli. It is plausible that listening to action verbs pronounced with different vitality forms evokes in the participants an internal simulation activating in them the same areas involved in the pronunciation of those action verbs except for the primary motor cortex. This hypothesis has been tested in a subsequent fMRI experiment showing that in the dorso-central insula are present voxels selective for both listening and imaging speech vitality forms. These findings strongly suggest the existence of the same neural substrate located in the insula which is involved in the perception and expression of speech vitality forms [7].

The activity of the insula in response to auditory stimuli endowed with vitality forms is in agreement with previous fMRI studies described above on action vitality forms $[3,6]$. These data corroborate the idea that the central sector of the insular cortex is the key region for vitality forms processing. During social interactions, this area is triggered not only by action vitality forms (observation, imagination, execution) but also by speech vitality forms (listening, imagination) indicating that the dorsocentral insula plays a crucial role in the processing of vitality forms regardless of the modality with which they are conveyed.

\subsection{The Role of Vitality Forms in Social Interactions}

In everyday life, people socially interact expressing their positive or negative attitudes by performing actions or pronouncing words. The expression of vitality forms allows individuals to communicate their own internal state while the perception of vitality forms allow them to understand those of others. For example, if actions or words are performed/pronounced gently or rudely, the receiver can understand if the agent is angry or calm. It is still unknown whether, during social interactions, gentle and rude vitality forms expressed by the agent may influence positively or negatively the motor behavior of the receiver. In this regard, a kinematic study was carried out to investigate whether and how two action requests (give me; take it) performed by an actor or an actress with different vitality forms (rude and gentle) may affect the kinematics of a subsequent motor response performed by participants [5]. Fourteen right-handed participants took part in the study. For each participant, a reflective marker was placed on the nails of right thumb, the index finger (grasping markers) and on the wrist (reaching marker). The two grasping markers allowed to record the grasping phase of the action characterized by an initial phase of fingers opening up to a maximum (maximal finger aperture), followed by a phase of the finger closing on 
the object. Differently, the reaching marker allowed to analyze the kinematics of the reaching phase. During the experiment, participants were presented with video clips showing an actor/actress performing a giving request (asking for a bottle; task 1, Fig. 6.8a) or a taking request (handing a bottle; task 2, Fig. 6.8b). Most importantly, each request was presented as visual action (V: visual modality) or auditory action verb (A: auditory modality) or both (AV: audio-visual) (Fig. 6.8). All the requests were expressed with rude and gentle vitality forms. After the actor's request (V, A, $\mathrm{AV}$ ), participants performed a subsequent action (reach-to-grasp the bottle with the goal to give or to take it).

The results indicated that, for both tasks (giving action, taking action), the perception of vitality forms modulated the kinematic parameters (velocity and trajectory) of the subsequent action performed by participants. In particular, concerning the reaching phase (Fig. 6.9a, b), vitality forms modulated the temporal (acceleration and velocity) and spatial parameters (trajectory) of the reach component, showing a wider trajectory and higher velocity in response to the rude requests compared to the gentle ones. Additionally, concerning the grasping phase (Fig. 6.9c, d), results showed a wider maximal finger aperture in response to rude vitality form than the gentle one. Taken together, these data indicate that vitality forms expressed by the actors influenced both the reach and grasp components of the motor acts performed by participants.

It is important to note that the effect of vitality forms expressed by the actor/actress on the motor response of the receiver also occurred when participants simply heard the verbal requests pronounced gently or rudely. This suggests that the influence of vitality forms on the participants' motor responses cannot be merely ascribed to an imitation mechanism of the observed actions. During the perception of vitality forms, the physical parameters characterizing actions execution (velocity, trajectory) or words pronunciation (pitch, intensity) are encoded in the dorso-central insula. The

A TASK 1

B TASK 2
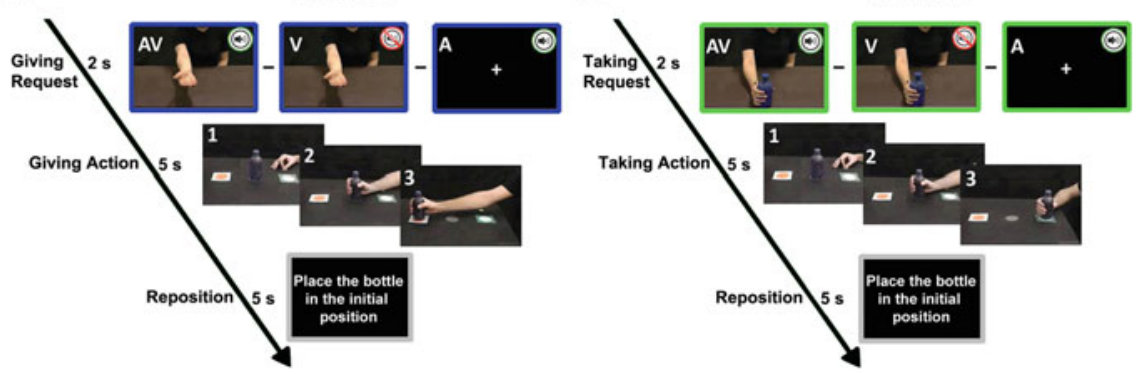

Fig. 6.8 Experimental paradigm. Participants were presented with audio-visual (AV), visual (V), and auditory (A) (a) stimuli. In the task 1, after the request, participants were requested to give the bottle (a). In the task 2, after the request, participants were requested to take the bottle (b). Panels with numbers display the phases of the participants' movement during the experimental trial: 1 , starting position; 2 , grasping the bottle; 3 , taking (or giving) the bottle. Time line reports the timing of different trial phases. Figure adapted from Di Cesare et al. [5] 


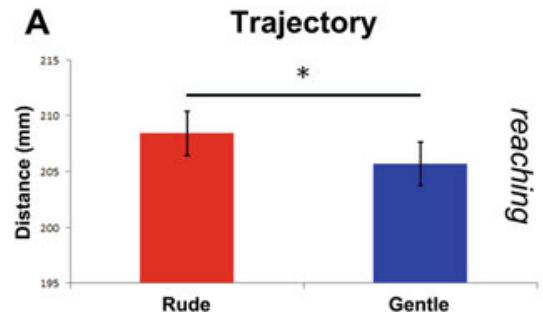

B

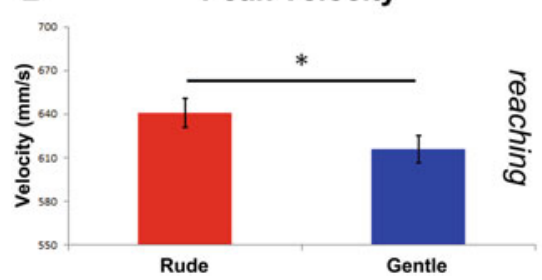

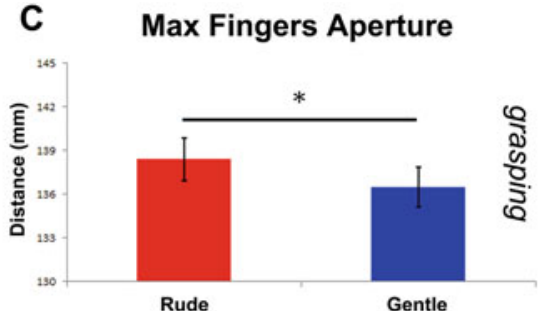

D Peak Velocity Closure

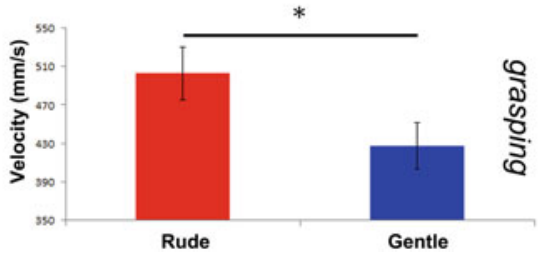

Fig. 6.9 Graphs display the mean values of kinematic parameters recorded in participants in response to a rude or gentle vitality forms during the reaching $(\mathbf{a}, \mathbf{b})$ and the grasping phases $(\mathbf{c}, \mathbf{d})$. Vertical bars represent the standard error of the mean (SEM). The asterisk (*) indicates the statistical significance $(p<0.05)$. Figure adapted from Di Cesare et al. [5]

role of the insula would be to transform the visual and acoustic information of the perceived vitality forms into a motor domain allowing the receiver to understand the positive or negative attitude of the agent and prepare the adequate motor response.

\subsection{Conclusions}

An important aspect of the action that characterizes human interactions is the vitality form. Vitality form represents the way in which actions and words are performed or pronounced. The expression of vitality forms allows people to communicate their attitudes while the perception of vitality forms allows them to understand those of others. This mechanism is important to relate to and understand others from a psychological point of view. The findings described in this chapter highlight the fundamental role of vitality forms in social communication and lay the foundations for future studies on human-human and human-robot interactions. The concept of vitality form could be used in the future operating systems of robots, which would allow them, on the one hand, to detect the positive or negative attitudes of humans, and, on the other hand, to assume the correct role in different contexts such as an authoritative role in the security context. From this perspective, vitality forms could become a future fundamental source of social communication to promote not only human-human interactions but also human-robot interactions. 
Acknowledgements Giuseppe Di Cesare is supported by a Starting Grant from the European Research Council (ERC) under the European Union's Horizon 2020 research and innovation program. G.A. No. 804388, wHiSPER.

\section{References}

1. Anderson, W., \& Jaffe, J. (1972). The definition and timing of vocalic syllables in speech signals. Scientific Reports, 12.

2. Caruana, F., Avanzini, P., Gozzo, F., Francione, S., Cardinale, F., \& Rizzolatti, G. (2015). Mirth and laughter elicited by electrical stimulation of the human anterior cingulate cortex. Cortex, $71,323-331$.

3. Di Cesare, G., Di Dio, C., Rochat, M. J., Sinigaglia, C., Bruschweiler-Stern, N., Stern, D. N., et al. (2013). The neural correlates of "vitality form" recognition: An fMRI study. Social Cognitive and Affective Neuroscience, 9(7), 951-960.

4. Di Cesare, G., Fasano, F., Errante, A., Marchi, M., \& Rizzolatti, G. (2016). Understanding the internal states of others by listening to action verbs. Neuropsychologia, 89, 172-179.

5. Di Cesare, G., De Stefani, E., Gentilucci, M., \& De Marco, D. (2017). Vitality forms expressed by others modulate our own motor response: A kinematic study. Frontiers in Human Neuroscience, 11, 565.

6. Di Cesare, G., Di Dio, C., Marchi, M., \& Rizzolatti, G. (2015). Expressing our internal states and understanding those of others. Procedure of the National Academic Science of the United States of America, 112(33).

7. Di Cesare, G., Marchi, M., Errante, A., Fasano, F., \& Rizzolatti, G. (2017). Mirroring the social aspects of speech and actions: The role of the insula. Cerebral Cortex, 1-10.

8. di Pellegrino, G., Fadiga, L., Fogassi, L., Gallese, V., \& Rizzolatti, G. (1992). Understanding motor events: A neurophysiological study. Experimental Brain Research, 91(1), 176-180.

9. Kriegeskorte, N., Simmons, W. K., Bellgowan, P. S., \& Baker, C. I. (2009). Circular analysis in systems neuroscience: The dangers of double dipping. Nature Neuroscience, 12(5), 535-540. https://doi.org/10.1038/nn.2303.

10. Kurth, F., Zilles, K., Fox, P. T., Laird, A. R., \& Eickhoff, S. B. (2010). A link between the systems: Functional differentiation and integration within the human insula revealed by metaanalysis. Brain Structure and Function, 214, 519-534.

11. Rizzolatti, G., Cattaneo, L., Fabbri-Destro, M., \& Rozzi, S. (2014). Cortical mechanisms underlying the organization of goal-directed actions and mirror neuron-based action understanding. Physiological Reviews, 94, 655-706.

12. Rizzolatti, G., \& Sinigaglia, C. (2016). The mirror mechanism: A basic principle of brain function. Nature Reviews Neuroscience, 12, 757-765.

13. Singer, T., Seymour, B., O’Doherty, J., Kaube, H., Dolan, R. J., \& Frith, C. D. (2004). Empathy for pain involves the affective but not sensory components of pain. Science, 303(5661), 11571162.

14. Stern, D. N. (1985). The interpersonal world of the infant. New York: Basic Books.

15. Stern, D. N. (2010). Forms of vitality exploring dynamic experience in psychology, arts, psychotherapy, and development. UK: Oxford University Press.

16. Wicker, B., Keysers, C., Plailly, J., Royet, J. P., Gallese, V., \& Rizzolatti, G. (2003). Both of us disgusted in my insula: The common neural basis of seeing and feeling disgust. Neuron, 40, $655-664$. 
Open Access This chapter is licensed under the terms of the Creative Commons Attribution 4.0 International License (http://creativecommons.org/licenses/by/4.0/), which permits use, sharing, adaptation, distribution and reproduction in any medium or format, as long as you give appropriate credit to the original author(s) and the source, provide a link to the Creative Commons license and indicate if changes were made.

The images or other third party material in this chapter are included in the chapter's Creative Commons license, unless indicated otherwise in a credit line to the material. If material is not included in the chapter's Creative Commons license and your intended use is not permitted by statutory regulation or exceeds the permitted use, you will need to obtain permission directly from the copyright holder.

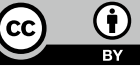

